\title{
CRIS: A new method in isomeric beam production
}

\author{
K.M. Lynch ${ }^{1,2, a}$, J. Billowes ${ }^{1}$, M.L. Bissell ${ }^{3}$, I. Budincevic ${ }^{3}$, T.E. Cocolios ${ }^{1}$, R.P. De Groote ${ }^{3}$, S. De Schepper ${ }^{3}$, V.N. \\ Fedosseev $^{4}$, K.T. Flanagan ${ }^{1}$, S. Franchoo ${ }^{5}$, R.F. Garcia Ruiz ${ }^{3}$, H. Heylen ${ }^{3}$, B.A. Marsh ${ }^{4}$, P.J.R. Mason ${ }^{6}$, G. Neyens $^{3}$, \\ T.J. Procter ${ }^{1}$, R.E. Rossel ${ }^{4,7}$, S. Rothe ${ }^{4,7}$, G.S. Simpson ${ }^{8}$, A.J. Smith ${ }^{1}$, I. Strashnov ${ }^{1}$, H.H. Stroke ${ }^{9}$, P.M.Walker ${ }^{6}$, \\ K.D.A. Wendt ${ }^{7}$, and R.T. Wood ${ }^{6}$ \\ ${ }^{1}$ School of Physics and Astronomy, The University of Manchester, Manchester, M13 9PL, United Kingdom \\ ${ }^{2}$ ISOLDE, PH Department, CERN, CH-1211 Geneva-23, Switzerland \\ ${ }^{3}$ Instituut voor Kern- en Stralingsfysica, KU Leuven, B-3001 Leuven, Belgium \\ ${ }^{4}$ EN Department, CERN, CH-1211 Geneva 23, Switzerland \\ ${ }^{5}$ Institut de Physique Nucléaire d'Orsay, F-91406 Orsay, France \\ ${ }^{6}$ Department of Physics, University of Surrey, Guildford GU2 7XH, United Kingdom \\ ${ }^{7}$ Institut für Physik, Johannes Gutenberg-Universität Mainz, D-55128 Mainz, Germany \\ ${ }^{8}$ LPSC, F-38026 Grenoble, France \\ ${ }^{9}$ Department of Physics, New York University, NY, New York 10003, USA
}

\begin{abstract}
The Collinear Resonance Ionization Spectroscopy (CRIS) experiment at ISOLDE, CERN, uses laser radiation to stepwise excite and ionize an atomic beam for the purpose of ultra-sensitive detection of rare isotopes, and hyperfine-structure measurements. The technique also offers the ability to purify an ion beam that is heavily contaminated with radioactive isobars, including the ground state of an isotope from its isomer, allowing decay spectroscopy on nuclear isomeric states to be performed. The isomeric ion beam is selected by resonantly exciting one of its hyperfine structure levels, and subsequently ionizing it. This selectively ionized beam is deflected to a decay spectroscopy station (DSS). This consists of a rotating wheel implantation system for alpha- and beta-decay spectroscopy, and up to three germanium detectors around the implantation site for gamma-ray detection. Resonance ionization spectroscopy and the new technique of laser assisted nuclear decay spectroscopy have recently been performed at the CRIS beam line on the neutron-deficient francium isotopes. Here an overview of the two techniques will be presented, alongside a description of the CRIS beam line and DSS.
\end{abstract}

\section{Introduction}

The Collinear Resonance Ionization Spectroscopy (CRIS) experiment [1] at ISOLDE, CERN, utilities the selectivity of resonance ionization to perform decay spectroscopy on pure nuclear isomeric states [2]. The novel technique of laser assisted nuclear decay spectroscopy in a collinear geometry combines hyperfine structure measurements with radioactive-decay measurements, providing additional information on the isotope, or isomer, under investigation.

An experimental campaign measuring the neutrondeficient francium isotopes is underway at CRIS. As the isotopes in this region of the nuclear chart (above the $\mathrm{Z}=82$ magic number) become more neutron-deficient, a decrease in the excitation energy of the $\left(\pi \mathrm{s}_{1 / 2}{ }^{-1}\right) 1 / 2^{+}$ level has been observed. In ${ }^{195} \mathrm{At}$ and ${ }^{185} \mathrm{Bi}$ it is this $\left(\pi \mathrm{s}_{1 / 2}{ }^{-1}\right) 1 / 2^{+}$deformed intruder state that is the ground state $[3,4]$. Recent in-beam radioactive decay measurements of ${ }^{201,203} \mathrm{Fr}$ provide evidence for the presence of a $\left(\pi \mathrm{s}_{1 / 2}{ }^{-1}\right) 1 / 2^{+}$isomeric intruder state, suggesting that ${ }^{199} \mathrm{Fr}$ has a $\mathrm{I}=1 / 2^{+}$ground state spin with an associated large

\footnotetext{
a e-mail: kara.marie.lynch@cern.ch
}

oblate deformation [5-7]. Through measurement of the hyperfine structure of the neutron deficient francium isotopes towards ${ }^{199} \mathrm{Fr}$, the spins, magnetic moments and change in mean-square charge radii of both the ground state and isomeric states will be investigated. This provides important information to better understand the evolution of nuclear structure in this region of the nuclear chart.

In addition, with the technique of laser assisted nuclear decay spectroscopy, alpha- and gamma-decay measurements can be performed on each isomeric beam separately. The previous decay spectroscopy of ${ }^{202} \mathrm{Fr}$ and ${ }^{204} \mathrm{Fr}$ respectively could not differentiate the alpha-decay patterns of the isomeric states from those of the ground state [8]. However, later measurements [9] revealed the low-lying structure of these isotopes, but the tentative spin-parity assignments of these isomers remain based on feeding patterns in $\beta+/$ EC decay and on systematics. By utilising the collinear resonance ionization method as a purification technique, isomeric beams can be studied, answering questions on the presence, lifetime and decay mechanism of each state. 


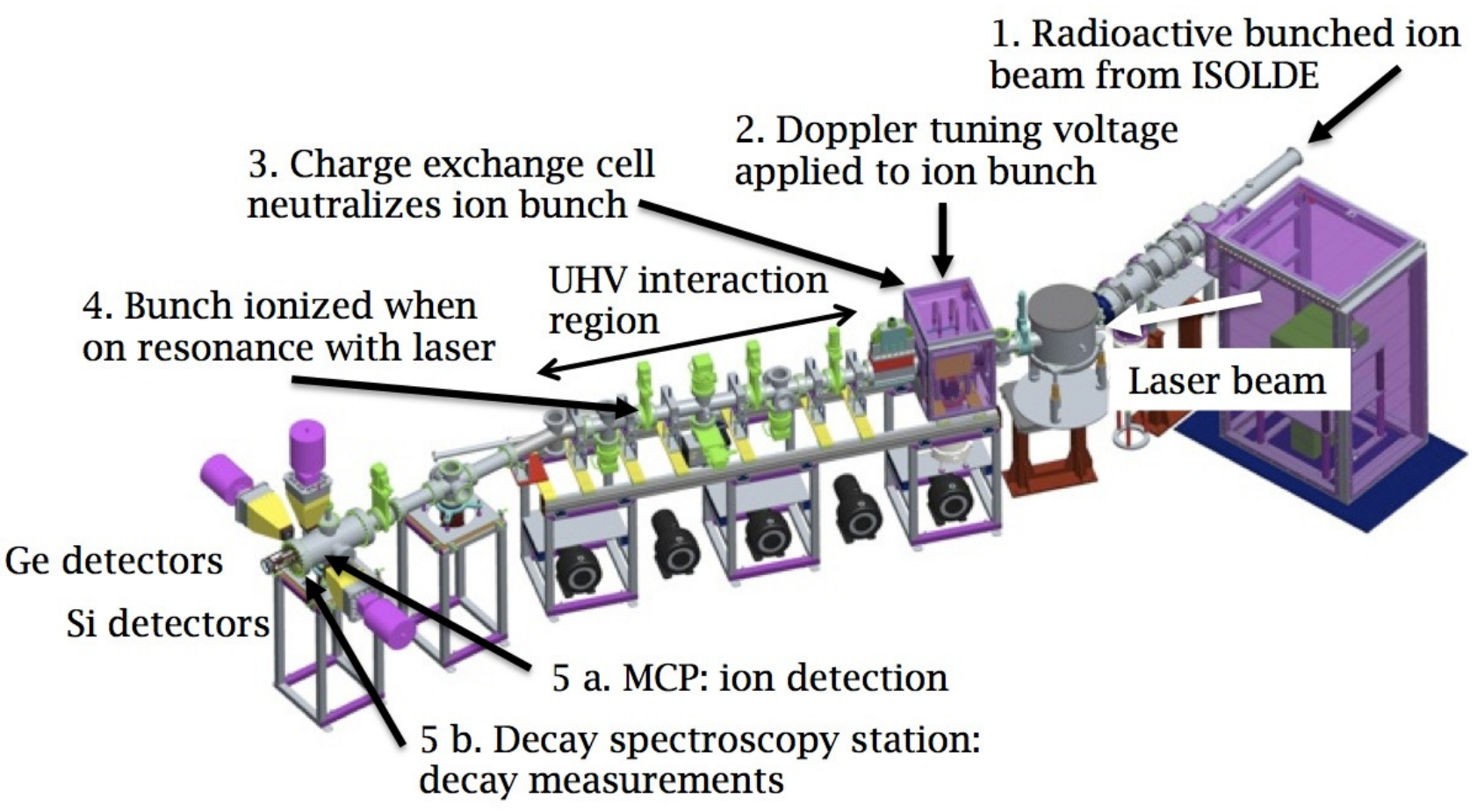

Figure 1. The CRIS beam line at ISOLDE, CERN, uses laser radiation to probe an isotope's hyperfine structure.

\section{Collinear resonance ionization spectroscopy}

Laser spectroscopy provides model-independent measurements of an isotope's nuclear spin, magnetic dipole moment, electric quadrupole moment and the change in mean-square charge radii between isotopes [10].

Scanning the frequency of the laser probes the isotope's hyperfine structure, and when the laser frequency is on resonance with an atomic transition, the isotope is resonantly ionized. This resonance ionization process selects the isotope of interest. The selectivity of an isotope is given by

$$
S=\prod\left(\frac{\Delta \omega_{A B, n}}{\Gamma_{n}}\right)^{2}=\prod S_{n},
$$

where $\delta \omega_{A B, n}$ is the separation in frequency of the two states, $\Gamma_{n}$ is the width of the resonance and $S_{n}$ is the selectivity of the $n$th transition. Thus, the higher the number of excitation steps, the greater the selectivity.

With the benefit of the high resolution inherent to collinear laser spectroscopy, CRIS can be used as a purification technique. Different isotopes and isomers have different nuclear observables, leading to unique hyperfine structures. By using laser radiation, it is possible to stepwise selectively excite and ionize an atomic beam in a particular isomeric state. Deflection of this selectively ionized beam, from the remaining neutral contaminants, allows ultra-sensitive detection of rare isotopes and nuclear structure measurements. Decay spectroscopy can therefore be performed on pure isomeric beams with a near complete suppression of the ground state.

\section{The CRIS beam line}

Francium isotopes are produced by impinging $1.4 \mathrm{GeV}$ protons upon a thick target $\left(\mathrm{UC}_{x}\right)$. These radioactive products are then surface ionized (on a hot rhenium surface), accelerated to $40 \mathrm{keV}$ and mass separated with the highresolution separator (HRS) to select the isotope of interest. The francium beam is bunched using the ISOLDE coolerbuncher ISCOOL, which is essential for reducing the duty cycle losses associated with the pulsed lasers [11].

The bunched ion beam is delivered into the CRIS beam line, shown in Fig. 1. It is passed through an alkali vapour charge exchange cell (CEC) where it is neutralized. The atomic bunch is collinearly overlapped with two laser beams in the interaction region. In this region, ultra-high vacuum (UHV) is needed $\left(\sim 10^{-9} \mathrm{mbar}\right)$. This is to reduce the background due to non-resonant ionization associated with collisions with gas molecules. The collinear geometry of the setup gives a reduction in the thermal Doppler broadening by a factor of $10^{3}$, improving resolution compared to in-source spectroscopy. This is due to the product of the velocity and velocity spread remaining constant under acceleration, decreasing the Doppler-broadened line width of the hyperfine transition to below its natural width at $30 \mathrm{kV}$. This leads to a greater selectivity between the ground and isomeric states of the nucleus, and a higher resolution and sensitivity of the nuclear observables.

The francium isotopes were resonantly excited from the $7^{2} \mathrm{~S}_{1 / 2}$ electronic orbital to the $8^{2} \mathrm{P}_{3 / 2}$ state with $422.7 \mathrm{~nm}$ light. This was provided by the RILIS team at ISOLDE using a Ti:Sa laser [12] and fibre-coupled through $35 \mathrm{~m}$ of multimode optical fibre into the CRIS beam line. The laser frequency was scanned to measure 
the hyperfine structure. The second step was from the $8^{2} \mathrm{P}_{3 / 2}$ state into the continuum using $1064 \mathrm{~nm}$ light produced by a broadband Nd:YAG laser next to the CRIS experimental setup. The ions produced by this resonant ionization were deflected from the remaining neutral beam and delivered to the Decay Spectroscopy Station (DSS). They can either be detected with an MCP for hyperfine structure measurements, or implanted into a carbon foil for decay measurements.

\subsection{The decay spectroscopy station}

The DSS [13] consists of a rotating wheel implantation system, based on the design from KU Leuven [14]. There are four Canberra Passivated Implanted Planar Silicon (PIPS) detectors for charged particle detection (alpha, electron, fission fragments), situated on either side of a carbon foil, into which the beam is implanted. The DSS 'windmill' system can be seen in Fig. 2(a). One PIPS detector is situated behind the carbon foil, and one annular PIPS detector (with an aperture diameter of $4 \mathrm{~mm}$ ) is placed in front. The beam is manipulated through a collimator in front of the annular detector (to protect against direct implantation into the silicon wafer) and through the aperture of the annular detector, where it is implanted into a carbon foil. An additional pair of PIPS detectors are located at $108^{\circ}$ away from the the on-axis pair. This allows an unperturbed measurement of longer lived radioactivedecay products. Up to three HPGe detectors can be placed around the implantation site for gamma-ray detection. The stainless-steel material of the DSS chamber (necessary for UHV conditions) causes an attenuation of $<92 \%$ of gamma rays at $60 \mathrm{keV}$. At energies $>590 \mathrm{keV},<18 \%$ of gamma rays are stopped by the steel wall of the chamber. Inclusion of the attenuation of the steel-wheel housing the carbon foils, an efficiency of $\sim 0.3 \%$ was determined at 1 $\mathrm{MeV}$ [13].

Beam tuning to the carbon foil proved difficult with a radioactive ion beam. Consequently, a Faraday cup was designed and installed in the location of one of the carbon foils, shown in Fig. 2(b). This plate (thickness $0.5 \mathrm{~mm}$, diameter $10 \mathrm{~mm}$ ) is electrically isolated from the steel wheel by PEEK rings and connected by a spot-welded Kapton cable attached to a rotatable $\mathrm{BNC}$ connection in the centre of the wheel. In addition to the Faraday cup, the collimator located in front of the annular PIPS detector was electrically connected. By measuring the current generated by the ion beam, it was used as a beam monitoring device. When it is not in use, it is electrically grounded to avoid charge build-up. These additions increased the total beam transmission efficiency of the CRIS beam line from $<10 \%$ to $>35 \%$.

The decay data is recorded event-by-event with a digital data acquisition system (XIA Digital Gamma Finder (DGF) modules). Alpha-gamma coincidences can be reconstructed off-line to identify a radioactive decay and reduce the background. Due to the $1064 \mathrm{~nm}$ laser light reflecting down the vacuum chambers of the beam line, the DGF parameters had to be set to offset the effect on the baseline of the signal from the PIPS detectors.
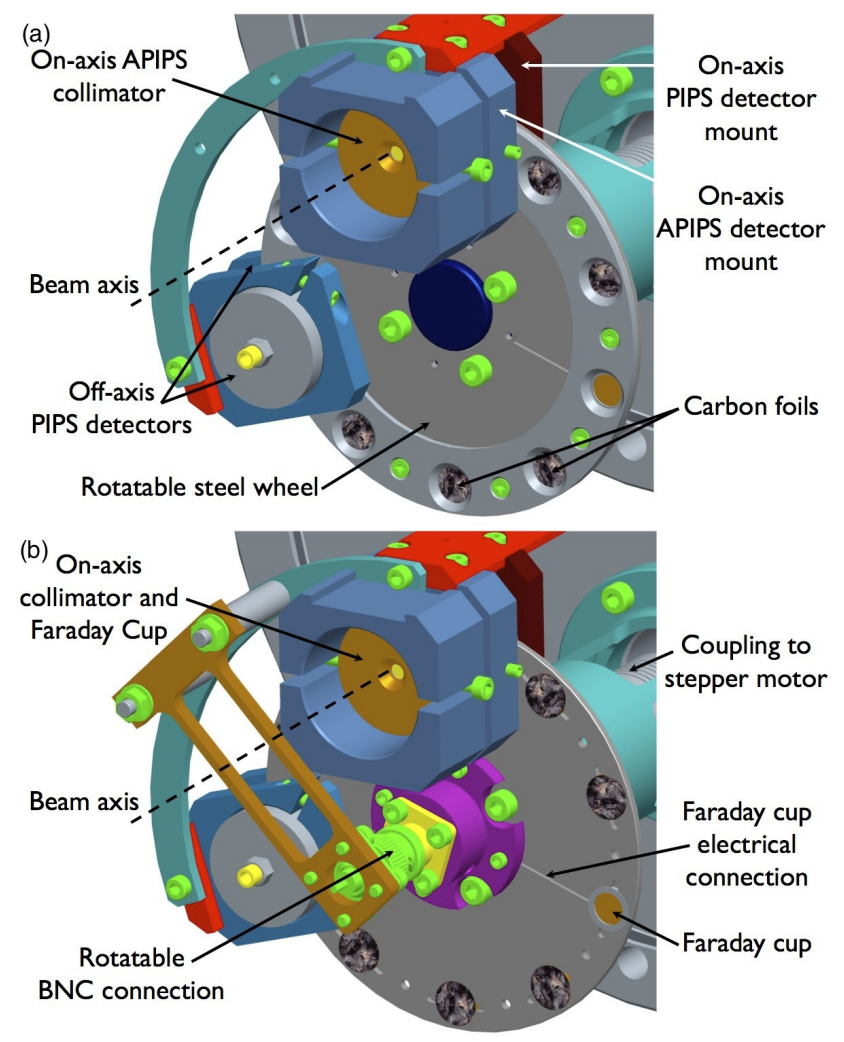

Figure 2. (a) The rotatable wheel and silicon detectors of the decay spectroscopy station 'windmill' system. (b) The newly installed Faraday cup. (Shown with carbon foil ring holder removed to highlight the Faraday cup.)

\subsection{Experimental efficiency}

The total experimental efficiency was measured to be 1 in 130 for ${ }^{202}$ Fr. This value includes the transmission, ionization, neutralization and detection efficiencies and was calculated from the yield of ${ }^{202} \mathrm{Fr}$. This yield was calculated in parallel from alpha-decay measurements. This efficiency increased to 1 in 60 for the isotope ${ }^{218} \mathrm{Fr}$, after the neutralization efficiency of the CEC increased towards the end of the experiment. The non-resonant ionization efficiency (calculated from the pressure in the interaction region and the cross-section of ionization) was between 1 in $10^{5}$ and 1 in $10^{6}$. This agrees well with the measured background suppression of 1 in $10^{5}$. The purity of the isomeric beams was measured to be $\sim 1$ in $10^{4}$.

\section{Hyperfine measurements with CRIS}

The CRIS experiment was successful in performing the first measurement of the hyperfine structure of the neutrondeficient francium isotopes ${ }^{202-206,218,219} \mathrm{Fr}$. The isotope ${ }^{221} \mathrm{Fr}$ was used as the reference isotope. An example scan of the hyperfine structure can be seen in Fig. 3.

The reference scans of ${ }^{221} \mathrm{Fr}$ were analysed to test the consistency of the CRIS results with literature. A comparison of the hyperfine parameter $A\left(\mathrm{~S}_{1 / 2}\right)$ from the reference scans with the established value of 6204.6(8) MHz [15] is shown in Fig. 4. The value agrees within errors, but 


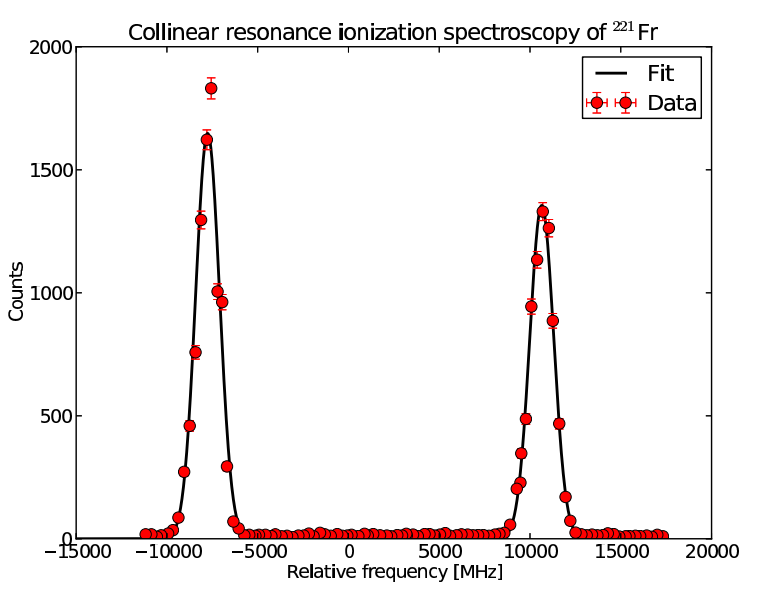

Figure 3. The hyperfine structure of ${ }^{221} \mathrm{Fr}$, measured with collinear resonance ionization spectroscopy.

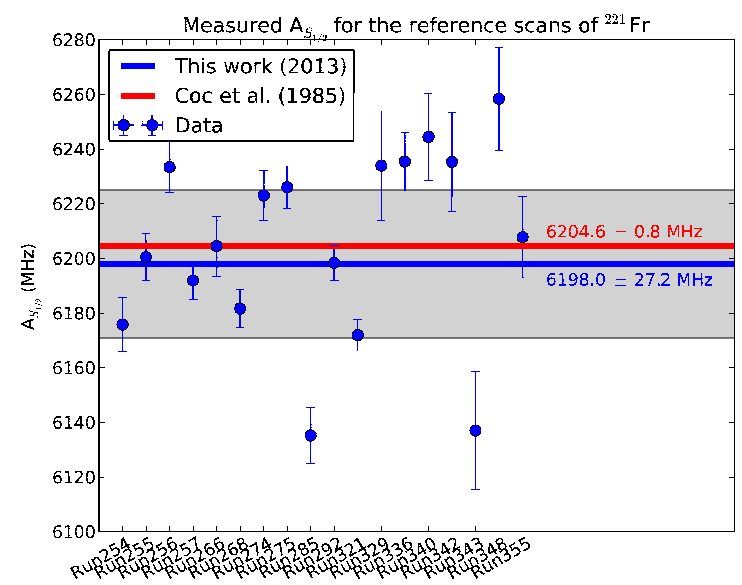

Figure 4. The spread of $A\left(\mathrm{~S}_{1 / 2}\right)$ values measured from the ${ }^{221} \mathrm{Fr}$ reference scans. The weighted mean values agrees well with the accepted literature value [15]. The grey region denotes one standard deviation away from the weighted mean.

highlights the scatter in $A\left(\mathrm{~S}_{1 / 2}\right)$ of the ${ }^{221} \mathrm{Fr}$ data. A significant contribution to this arises due to the variation in synchronization between the two laser pulses in the resonance ionization process.

\section{Laser assisted nuclear decay spectroscopy}

The novel technique of laser assisted nuclear decay spectroscopy [16] was successfully performed on ${ }^{202,204,218} \mathrm{Fr}$. The purification technique of collinear resonance ionization was employed to separate the three low-lying states of ${ }^{204} \mathrm{Fr}\left({ }^{204 g} \mathrm{Fr} \operatorname{spin} 33^{(+)},{ }^{204 m 1} \mathrm{Fr}\right.$ spin $\left(7^{+}\right)$and ${ }^{204 m 2} \mathrm{Fr}$ spin $\left(10^{-}\right)$), and nuclear-decay measurements on each were carried out. The measurement of the hyperfine structure of the beam of ${ }^{204 g, m 1, m 2} \mathrm{Fr}$ was enough to prove the existence of the three states, and consequent radioactive-decay studies performed on selectively ionized isomeric hyperfine com- ponents measured the energies of the alpha particles emitted from each state and their daughters.

\section{Conclusions and Outlook}

The CRIS technique uses the selectivity of resonance ionization to purify an ion beam contaminated with radioactive isobars, with the ability to separate a ground state from its isomer. From the resulting isomeric beam, pure decay spectroscopy can be performed. Hyperfine structure measurements have been made on the neutron-deficient francium isotopes ${ }^{202-206,218,219} \mathrm{Fr}$, with laser assisted nuclear decay spectroscopy having been performed for the first time on ${ }^{202,204,218}$ Fr.

\section{Acknowledgements}

The authors extend their thanks to the ISOLDE team for providing the beam, the GSI target lab for producing the carbon foils, IKS-KU Leuven and University of Manchester machine shops for their work, IKS-KU Leuven and the UK-France loan pool for the use of their Ge detectors. This work was supported by the IAP project P7/23 of the OSTC Belgium (BRIX network) and by the FWO-Vlaanderen (Belgium). The Manchester group was supported by the STFC grants ST/F012071/1 and ST/J000159/1. K.T. Flanagan was supported by STFC Advanced Fellowship Scheme grant number ST/F012071/1. The University of Surrey support was from STFC grant ST/J000051/1 and AWE plc. The authors would also like to thank Ed Schneiderman for continued support through donations to the Physics Department at NYU.

\section{References}

[1] T.J. Procter et al., Journal of Physics: Conference Series 381, 012070 (2012)

[2] K.M. Lynch et al., Journal of Physics: Conference Series 381, 012128 (2012)

[3] H. Kettunen et al., Eur. Phys. J. A 16, 457 (2003)

[4] C.N. Davids et al., Phys. Rev. Lett. 76, 592 (1996)

[5] J. Uusitalo et al., Phys. Rev. C 71, 024306 (2005)

[6] U. Jakobsson et al., Phys. Rev. C 85, 014309 (2012)

[7] Z. Kalaninová et al., Phys. Rev. C 87, 044335 (2013)

[8] P. Hornshoj et al., Nucl. Phys. A 230, 380 (1974)

[9] M. Huyse et al., Phys. Rev. C 46, 1209 (1992)

[10] K. Blaum et al., Physica Scripta 2013, 014017 (2013)

[11] E. Mané et al., Eur. Phys. J. A 42, 503 (2009)

[12] S. Rothe et al. (2013), doi: 10.1016/j.nimb.2013.08.058

[13] M.M. Rajabali et al., Nucl. Instrum. Methods Phys. Res. A 707, 35 (2013)

[14] A.N. Andreyev et al., Phys. Rev. Lett. 105, 252502 (2010)

[15] A. Coc et al., Physics Letters B 163, 66 (1985)

[16] K.M. Lynch et al., Hyperfine Interactions 216, 95 (2013) 